\section{Arbeitszeitwünsche von Frauen und Männern}

Forschungsergebnisse des DIW Berlin für den Untersuchungszeitraum 1993 bis 2005 zeigen, dass die Arbeitszeitwünsche und die vereinbarten Arbeitszeiten von Frauen und Männern weit näher beieinander liegen als ihre tatsächlichen Wochenarbeitszeiten. In Deutschland liegen die von abhängig Beschäftigten gewünschten Wochenarbeitszeiten im Durchschnitt zwischen etwa 30 Stunden (Frauen) und 39 Stunden (Männer). Dieser geschlechtsspezifische Abstand („gender gap“) ließe sich auf acht Stunden bei einer Realisierung der vertraglich vereinbarten Wochenarbeitszeit reduzieren. Tatsächlich sind Männer 2005 jedoch mit 42,2 Stunden im Durchschnitt rund elf Wochenstunden länger erwerbstätig als Frauen. Hier ist auch die in den amtlichen Statistiken nicht erfasste unbezahlte Mehrarbeit von Beschäftigten berücksichtigt.

Die meisten Beschäftigten wünschen sich Standard-Wochenarbeitszeiten wie 20,30, 35 oder 40 Wochenarbeitsstunden. Sehr lange Wochenarbeitszeiten von über 40 Stunden sind weder für eine Mehrheit von Frauen noch für Männer attraktiv. Allerdings klaffen hier Wunsch und Wirklichkeit stark auseinander, denn 52\% der erwerbstätigen Männer sind 41 und mehr Wochenstunden tätig (Frauen 16\%) aber nur $16 \%$ wünschen dies auch (Frauen $4 \%)$. Über die Hälfte der Frauen (54\%, 12\% der Männer) möchte weniger als 35 Stunden wöchentlich arbeiten. Wochenarbeitszeiten von weniger als 20 Stunden werden von den Frauen seltener gewünscht (14\%) als tatsächlich ausgeübt (19\%); bei den Männer ist es umgekehrt (7\% bzw. 3\%).

\section{Forschungsinitiative Diabetes}

Die Zahl der an Diabetes erkrankten Menschen steigt von Jahr zu Jahr. Mittlerweile sind rund 8\% der
Deutschen betroffen. Besonders alarmierend ist die Zahl der erkrankten Kinder und Jugendlichen. Um die Forschung in diesem Bereich deutlich zu stärken, fördert das Bundesministerium für Bildung und Wissenschaft (BMBF) zukünftig zwei krankheitsbezogene Kompetenznetze, für die in den nächsten zehn Jahren rund 100 Millionen Euro vorgesehen sind.

Wissenschaftlerinnen und Wissenschaftler sind nun aufgerufen, bis zum 17. September 2007 ihre Projektskizzen zu Forschungsvorhaben einzureichen.

Weitere Informationen stehen im Internet unter www.bmbf.de/foerderungen/8003.php (Adipositas) www.bmbf.de/foerderungen/8004.php (Diabetes)

\section{Selbstständig \& Gesund}

Selbstständig Erwerbstätige sind für die wirtschaftliche Entwicklung und für den Arbeitsmarkt von zunehmender Bedeutung. Allerdings fallen sie durch das Netz des Arbeitsschutzsystems. Zwar sind sie für die Sicherheit und Gesundheit ihrer Beschäftigten bei der Arbeit verantwortlich, ihr eigener Präventionsbedarf bleibt jedoch außen vor. Dies trifft auch für die zahlreichen „Solo-Selbstständigen" zu. Dabei sind Selbstständige mindestens so großen Belastungen ausgesetzt wie Beschäftigte. Erschwerend kommen die Unsicherheit der Marktsituation sowie die hohe Eigenverantwortung hinzu.

Die Bundesanstalt für Arbeitsschutz und Arbeitsmedizin (BAuA) hat jetzt das Projekt „Selbstständig \& Gesund“ abgeschlossen, das sie im Auftrag des Bundesministeriums für Arbeit und Soziales (BMAS) durchgeführt hat. Dabei entstanden Vorschläge für ein nationales Handlungskonzept, um den Arbeits- und Gesundheitsschutz für Selbstständige zu verbessern.

Das Projekt durchlief drei zentrale Entwicklungsschritte. Auf der Basis einer Bestandsaufnahme wurden Empfehlungen entwickelt, aus denen ein Vorschlag für ein natio- nales Handlungskonzept abgeleitet werden konnte. Die erarbeiteten Empfehlungen setzen Schwerpunkte im Bereich persönliche Prävention, gendersensible Prävention, Ausbau etablierter Systeme der Ansprache und Existenzgründer-Unterstützung. Auf dieser Basis kann Deutschland eine qualifizierte Empfehlung an die Europäische Union abgeben.

Weitere Informationen stehen im Internet unter www.selbststaendig-und-gesund.de.

\section{Mikrodaten von Krankenversicherten}

Ab sofort stellt das Statistische Bundesamt der Wissenschaft Mikrodaten von mehr als 2 Millionen Versicherten der gesetzlichen Krankenversicherung (GKV) für das Jahr 2002 zur Verfügung. Die formal anonymisierten Daten können über den Weg der kontrollierten Datenfernverarbeitung genutzt werden. Bei dem umfangreichen Datenmaterial handelt es sich um eine 3\%-Zufallsstichprobe von Versicherten der gesetzlichen Krankenversicherung mit Informationen über ambulante und stationäre Diagnosen, verordnete Arzneimittel, Krankengeldbezug sowie Arbeitsunfähigkeit aus dem Jahr 2002.

Das Statistische Bundesamt hat Daten aus einer wissenschaftlichen Untersuchung aufbereitet, die im Zuge der Reform des Risikostrukturausgleiches (RSA) in der gesetzlichen Krankenversicherung durchgeführt wurde. Die Untersuchungsergebnisse der beauftragten Forschungsinstitute wurden im November 2004 vorgelegt.

Auswertungen können über die kontrollierte Datenfernverarbeitung durchgeführt werden. Auf diesem Weg können Einzeldaten mittels selbst entwickelter Programme genutzt werden. Dabei wird ein Auswertungsprogramm an das Forschungsdatenzentrum des Statistischen Bundesamtes geschickt und dort auf die formal anonymisierten Daten angewendet. Anträge zur Nutzung der vorliegenden Daten mittels kontrollierter Datenfernverarbeitung für Vorhaben der unabhängigen wissenschaftlichen 
Forschung können beim Statistischen Bundesamt gestellt werden.

Antragsformulare sowie weitere Informationen stehen im Internet unter www. forschungsdatenzentrum.de

\section{Leuchtturmprojekt Demenz}

Das Bundesgesundheitsministerium hat den Forschungsbericht „Rahmenempfehlungen zum Umgang mit herausforderndem Verhalten bei Menschen mit Demenz in der stationären Altenhilfe" veröffentlicht. In Deutschland leben heute rund 1,1 Millionen Menschen, die an Demenz erkrankt sind. Bis zum Jahr 2030 wird sich diese Zahl auf ca. 1,7 Millionen erhöhen. Durch gezielte Maßnahmen kann das Fortschreiten der Krankheit jedoch in einem begrenzten Umfang aufgehalten und die Situation der Betroffenen verbessert werden. Durchgeführt wurde das Forschungsprojekt von einem Verbund des Instituts für Pflegewissenschaften der Universität Witten/Herdecke in Witten mit dem Kuratorium Deutsche Altershilfe (KDA), einer Beratungs- und Forschungsgesellschaft für Altenhilfe in Köln.

Verhaltensformen wie Aggressivität, Agitation, Apathie/Rückzugsverhalten und vokale Störungen sind sowohl für die Pflegeheimbewohner als auch für die Pflegenden mit besonderen Belastungen verbunden. Im Forschungsbericht werden Rahmenempfehlungen zu den folgenden Themen formuliert:

- Verstehende Diagnostik

- Assessmentinstrumente

- Validieren

- Erinnerungspflege

- Berührung/Sensorische Stimulation

- Bewegungsförderung

- und pflegerisches Handeln in akuten psychiatrischen Krisen.

Die Rahmenempfehlungen sind damit eine fundierte Grundlage für die zukünftige Entwicklung von Expertenstandards zur weiteren Qualitätssicherung in der Pflege.

Der Forschungsbericht steht im Internet unter www.bmg.bund.de Forschungsberichte

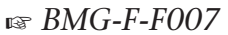

\section{"Move Europe" bewegt Mitarbeiter}

In immer mehr Führungsetagen setzt sich die Erkenntnis durch: Gesunde Mitarbeiter in gesunden Unternehmen sind eine wichtige Voraussetzung für Unternehmenserfolg. So werden allein in Deutschland jährlich 440 Millionen Arbeitsunfähigkeitstage verzeichnet. Die Kosten für diese Fehlzeiten belaufen sich auf etwa 35 Milliarden Euro. Ein Teil hiervon könnte durch ein stärkeres Investment in die betriebliche Gesundheitsförderung eingespart werden. So belegen Studien, dass mit jedem in betriebliche Gesundheitsförderung investierten Euro Krankheitskosten zwischen 2,50 und 4,80 Euro eingespart werden können.

"Move Europe" ist eine Initiative des Europäischen Netzwerks zur betrieblichen Gesundheitsförderung (ENWHP). Die Gesundheit europäischer Arbeitnehmer steht im Mittelpunkt. Die Kampagne, an der sich Unternehmen aus 27 Ländern beteiligen, läuft zunächst bis 2009. Der BKK Bundesverband koordiniert die Kampagne sowohl in Deutschland als auch europaweit. Interessierte Unternehmen können zunächst den Fragebogen zur Selbstbewertung als ersten Schritt auf dem Weg zum jährlich vergebenen „Unternehmenspreis Gesundheit" ausfüllen. Um den Preis bewerben können sich Unternehmen mit einem überzeugenden Gesundheitsmanagement erstmals bis zum 31. Oktober 2007.

Weitere Informationen stehen im Internet unter www.move-europe.de

\section{Alkohol - reden wir drüber!}

Um Eltern zu unterstützen, hat die Bundeszentrale für gesundheitliche Aufklärung (BZgA) einen Ratgeber entwickelt, der Müttern und Vätern Wege für das Gespräch mit ihren Kindern aufzeigt. Die neue Broschüre mit dem Titel „Alkohol - reden wir drüber!" berücksichtigt die verschiedenen Altersstufen der Heranwach- senden und enthält praktische Informationen für den Gesprächsalltag. Sie enthält Regeln für die Eltern im Hinblick auf ihren eigenen Alkoholkonsum, informiert über gesundheitliche Auswirkungen von Alkohol auf den jugendlichen Körper, berichtet über die wesentlichen Inhalte des Jugendschutzgesetzes und gibt Tipps, wie Eltern mit ihren Kindern auch in problematischen Situationen im Gespräch bleiben können.

Die Broschüre empfiehlt, den Zugang zu Alkohol für Heranwachsende möglichst lange hinauszuzögern, sinnvolle Alternativen zum Alkoholkonsum anzubieten und die gesundheitlichen Risiken gerade für junge Menschen aufzuzeigen.

„Alkohol - reden wir drüber!“ - Ein Ratgeber für Eltern steht im Internet unter

www.bzga.de Infomaterialien/Bestellung Suchtvorbeugung (Bestellnummer 32101900)

\section{Bericht zur psychi- schen Gesundheit}

Der Berufsverband Deutscher Psychologinnen und Psychologen (BDP) hat in diesem Jahr erstmals einen Bericht zur psychischen Gesundheit der Nation vorgelegt. Im Mittelpunkt des diesjährigen Reports stehen Kinder, ihre Entwicklungsbedingungen und ihre psychische Gesundheit sowie die Schwachstellen in Prävention und Versorgung.

5\% der Kinder und Jugendlichen in Deutschland werden im Bericht des BDP aufgrund besonders ungünstiger Entwicklungsverläufe als chronisch psychisch beeinträchtigt eingestuft. Das sind 320.000 junge Menschen. Die gleiche Zahl von Kindern und Jugendlichen wird vom BDP als dringend behandlungsbedürftig eingeschätzt. Der Bericht, der auf der wissenschaftlichen Arbeit an mehreren Universitäten und der Auswertung der Erfahrungen von Praktikern basiert, stellt eine zum Teil erhebliche Unterversorgung auf diesem Gebiet fest. Auch die Qualität und Verfügbarkeit von Unterstützungssystemen für Schüler, Eltern und Lehrer, auf die 
in den Siegerländern der Bildungsstudie großer Wert gelegt wird, hält der BDP in Deutschland für dringend verbesserungsbedürftig.

\section{Patienteninformation „Chronisch obstruk- tive Lungenerkran- kung"}

Der Gemeinsame Bundesausschuss (G-BA) hat auf seiner Internetseite eine Patienteninformation zum Thema „Chronisch obstruktive Lungenerkrankungen (COPD)“ veröffentlicht. Die Artikel und Merkblätter sollen Patienten auf der Basis zuverlässiger Forschungsarbeiten über Diagnose-, Behandlungs- und Unterstützungsmöglichkeiten informieren. Ergänzt wurde diese Patienteninformation um ein Glossar.

Die COPD ist eine recht verbreitete Krankheit und tritt sogar häufiger auf als Asthma. Etwa jeder zehnte bis zwanzigste Erwachsene über 40 Jahre hat COPD. Das Hauptmerkmal dieser Krankheit ist ein chronischer Hustenreiz.

Weitere Informationen stehen im Internet unter www.g-ba.de Informations-Archiv Patienteninformationen

\section{Medikamenten- abhängigkeit}

In Zusammenarbeit mit der Arzneimittelkommission der deutschen Ärzteschaft und Fachleuten des Suchthilfesystems sowie Vertretern von Patientenorganisationen ist der Leitfaden „Medikamente - schädlicher Gebrauch und Abhängigkeit" erarbeitet worden und soll zu einer frühen Erkennung von suchtgefährdeten Patientinnen und Patienten beitragen. Ärztinnen und Ärzten kann er eine Hilfestellung bei der Behandlung einer bereits bestehenden Medikamentenabhängigkeit geben.

Jeder 6. erwachsene Bundesbürger versucht mindestens einmal pro Woche mit einem Medikament sein Befinden zu verbessern. Dazu gehören insbesondere Schlaf-, Beruhigungsund Schmerzmittel. Der oft schleichende Prozess einer Abhängigkeit wird von der Außenwelt kaum bemerkt. Experten schätzen, dass inzwischen etwa 1,5 Millionen Menschen in Deutschland medikamentenabhängig sind. Mit dem Alter steigt die Gefährdung; Frauen sind doppelt so häufig betroffen wie Männer.

Der Leitfaden steht im Internet unter www.bundesaerztekammer.de/downloads/Leitfaden_Medikamente_Vorabfassung.pdf

\section{Seltene Erkrankungen}

Rund vier Millionen Menschen in Deutschland leiden an so genannten seltenen Erkrankungen, europaweit sind es rund 20 Millionen Menschen. Eine gezielte Bündelung von Forschung in Europa soll nun durch das europäische Netzwerk E-RARE und seine neue Förderinitiative erfolgen. Das Netzwerk E-RARE wird künftig die Forschungsaktivitäten von fünf europäischen Ländern zu seltenen Krankheiten koordinieren.

Leiden beispielsweise unter einer Erkrankung in einem Land wenige hundert Patienten, ist es hilfreich, wenn Mediziner sich europaweit über Krankheitsverläufe austauschen, gemeinsame Datenbanken aufbauen, Studien mit größeren Patientenzahlen durchführen und gemeinsam neue Therapien entwickeln. Mit ERARE, für das die beteiligten Länder insgesamt zunächst 12,8 Millionen Euro für 3 Jahre ausgeben, ergänzt das Bundesministerium für Bildung und Forschung (BMBF) seine laufende nationale Förderung von Netzwerken für seltene Erkrankungen. Seit 2003 stellt das BMBF dafür 30 Millionen Euro zur Verfügung.

Weitere Informationen zur aktuellen Ausschreibung E-RARE stehen im Internet unter: www.gesundheitsforschung-bmbf.de/ de/1556.php

Weitere Informationen zur BMBFFörderung zu seltenen Erkrankungen stehen im Internet unter www.gesundheitsforschung-bmbf.de/ de/131.php

\section{Gesundheitsausgaben 2005}

Nach Berechnungen des Statistischen Bundesamtes beliefen sich die Ausgaben für Gesundheit im Jahr 2005 auf insgesamt 239,4 Milliarden Euro oder 10,7\% des Bruttoinlandsproduktes. Dies war im Vergleich zum Jahr 2004 ein Plus von 5,6 Milliarden Euro oder 2,4\%, nachdem die Gesundheitsausgaben 2004 nahezu stagnierten (+ 0,0\%). Die Ausgaben je Einwohner lagen bei 2.900 Euro (2004: 2.830 Euro).

Mit rund 57\% der Ausgaben war die gesetzliche Krankenversicherung der größte Ausgabenträger im Gesundheitswesen. Ihre Ausgaben erreichten 135,9 Milliarden Euro und lagen im Jahr 2005 um 3,3\% über denen des Vorjahres. Die privaten Haushalte und privaten Organisationen ohne Erwerbszweck zahlten 32,4 Milliarden Euro und damit 1,2\% mehr als im Jahr 2004. Ihr Anteil an den Gesamtausgaben betrug knapp 14\%. Gut 9\% oder 22,0 Milliarden Euro entfielen auf die private Krankenversicherung, deren Aufwendungen um $4,2 \%$ und damit deutlich überdurchschnittlich gewachsen waren.

Über die Hälfte der Gesundheitsausgaben entfiel auf Waren (das sind Arzneimittel inklusive Verbandmittel, Hilfsmittel, Zahnersatz und sonstiger medizinischer Bedarf) und ärztliche Leistungen. Für beide Leistungsarten wurden jeweils knapp 27\% der Gesamtausgaben aufgewendet. Während die Ausgaben für ärztliche Leistungen nur um 0,5\% im Vergleich zum Jahr 2004 gestiegen sind, wuchsen die Ausgaben für Waren um $5,4 \%$. Hauptverantwortlich für diesen überdurchschnittlichen Anstieg waren die Arzneimittel, für die 10,2\% mehr aufgewendet wurden als 2004.

Fast jeder zweite Euro der Gesamtausgaben wurde in Einrichtungen der ambulanten Gesundheitsversorgung ausgegeben (48\%). Die Ausgaben in diesen Einrichtungen stiegen im Vergleich zum Vorjahr um 2,6\%. Im (teil-)stationären Sektor wurden mit 87,4 Milliarden Euro 2,3\% mehr aufgewendet. 


\section{Online-Datenbank für Senioreninitiativen}

In der von der Bundesarbeitsgemeinschaft Seniorenbüros im Auftrag des Bundesministeriums für Familie, Senioren, Frauen und Jugend aufgebauten Datenbank sind inzwischen 1.200 Verbände und Organisationen zu finden. In der Datenbank können sich Senioren über Angebote und Möglichkeiten direkt vor ihrer Haustür informieren. Über die Suchfunktion lässt sich zum Beispiel ganz gezielt nach Aktivitäten, Dachverbänden oder Zielgruppen forschen: von einer Übersicht der Öffnungszeiten im Stadtmuseum über den Großelterndienst, die Angebote der Seniorenakademie bis hin zu den Tafeln für Bedürftige. Die Initiativen stellen ihre freiwilligen Mitarbeiterinnen und Mitarbeiter vor und stehen als Ansprechpartner zur Verfügung, auch für andere Initiativen. Wichtige Ansprechpartner für diese und ähnliche Initiativen sind auch die Seniorenbüros, die es inzwischen in 200 Kommunen in Deutschland gibt.

Weitere Informationen stehen im Internet unter www.senioren-initiativen.de

\section{Neues Wohnen im Alter}

Zahlreiche Städte und Landkreise wünschen sich Hilfe bei der Bewältigung der Herausforderungen, vor denen unsere älter werdende Gesellschaft steht. Das ergab eine Befragung des Kuratoriums Deutsche Altershilfe im Auftrag des Bundesministeriums für Familie, Senioren, Frauen und Jugend. Mit dem bundesweiten Modellprogramm „Neues Wohnen - Beratung und Kooperation für mehr Lebensqualität im Alter" bietet das Bundesfamilienministerium den Kommunen in der Praxis erprobte Wege an, wie sie den demografischen Wandel besser meistern können. Die Initiative richtet sich zum Beispiel an Stadt- und Sozialplaner, Altenhilfeträger sowie Nachbarschafts- und Selbsthilfeinitiativen.

Dass großer Beratungsbedarf bei den Kommunen besteht, hat die Befragung ergeben, an der sich 249 Städte und Landkreise beteiligt haben. Sie repräsentieren rund 51 Millionen Einwohnerinnen und Einwohner, darunter mehr als zehn Millionen ältere Menschen. Die Befragung ergab, dass die Kommunen im Schnitt zwar eine gute Wohnversorgung gewährleisten können. Doch vor allem in ländlichen Regionen ist das Angebot nicht groß genug, um älteren Menschen auch im Fall von Pflegebedürftigkeit Wohnmöglichkeiten zu bieten, die Lebensqualität und Teilhabe ermöglichen.

Die Studie „Wohnen im Alter - Ergebnisse einer bundesweiten Befragung der Landkreise und kreisfreien Städte" steht im Internet unter www.bmfsfj.bund.de Publikationen Ältere Menschen

\section{Berufsqualifikationen der Heilberufe in der EU}

Das Bundeskabinett hat den Gesetzentwurf zur Umsetzung der Richtlinie 2005/36/EG des Europäischen Parlaments und des Rates über die Anerkennung von Berufsqualifikationen der Heilberufe beschlossen. Die Änderungen dienen der Umsetzung der Richtlinie in nationales Recht. Dabei geht es um Heilberufe, für deren Ausbildung der Bund zuständig ist (z. B. Ärzte, Apotheker, Physiotherapeuten, Pflegeberufe etc.).

Die Richtlinie regelt $u$. a., dass Berufsgruppen mit einer Ausbildung in Heilberufen in den EU-Staaten gegenseitig anerkannt werden. Außerdem beinhaltet die Richtlinie Erleichterungen für Leistungserbringer, die nur vorübergehend und gelegentlich in einem anderen Mitgliedstaat arbeiten wollen. Sie müssen kein formelles Anerkennungsverfahren durchlaufen. Der Entwurf berücksichtigt außerdem die Regelungen, die im Zuge des Beitritts von Bulgarien und Rumänien zur EU erforderlich sind.

Die Umsetzung der Richtlinie hat bis zum 20. Oktober $2007 \mathrm{zu}$ erfolgen. Dies wird durch den vorliegenden Gesetzentwurf im Bereich des Bundes für die Heilberufe sichergestellt.

Der Gesetzestext steht im Internet unter:

www.bmg.bund.de Gesetze und

Verordnungen Gesetzes- und Verordnungsentwürfe

\section{Sicherheit am Arbeitsplatz}

Die EU-Kommission will die Gesundheit und Sicherheit am Arbeitsplatz weiter erhöhen. Im Rahmen einer neuen 5-Jahres-Strategie sollen Berufskrankheiten und Arbeitsunfälle bis 2012 EU-weit um 25\% reduziert werden. Die geplanten Maßnahmen umfassen vor allem bessere Information und Schulung, Wissensaustausch und die Integration von Gesundheit und Sicherheit am Arbeitsplatz in andere nationale und europäische Politikbereiche.

Die neue Strategie umfasst Maßnahmen auf europäischer und nationaler Ebene. Die EU-Kommission plant Sensibilisierungskampagnen und bessere Information und Schulung sowie eine Verbesserung und Vereinfachung des geltenden Rechts. Zudem soll Gesundheit und Sicherheit am Arbeitsplatz in andere nationale und europäische Politikbereiche wie Bildung und Forschung integriert werden. Nationale Strategien sollten sich auf die am meisten betroffenen Wirtschaftszweige konzentrieren.

Seit 2002 wurden bereits eine Verringerung von $17 \%$ der tödlichen Unfälle und ein Rückgang von 23\% bei Unfällen erreicht, die einen Arbeitsausfall von drei oder mehr Tagen zur Folge haben. Doch sind die Fortschritte ungleich über die einzelnen Länder, Wirtschaftszweige, Unternehmen und Arbeitnehmerkategorien verteilt.

Weitere Informationen stehen im Internet unter http://ec.europa.eu/employment_social/ health_safety/index_de.htm 